\title{
Evaluation of Compact Boundary of Outage Probability in HetNet
}

\author{
Senlin Jiang $\mathbb{D}^{1}$ and Jiamei Zhang ${ }^{2}$ \\ ${ }^{1}$ Wuxi Institute of Technology, Wuxi, China \\ ${ }^{2}$ Wuxi Institute of Communications Technology, Wuxi, China \\ Correspondence should be addressed to Senlin Jiang; jiangsl@wxit.edu.cn
}

Received 5 August 2021; Accepted 24 August 2021; Published 14 September 2021

Academic Editor: Zhihan Lv

Copyright (c) 2021 Senlin Jiang and Jiamei Zhang. This is an open access article distributed under the Creative Commons Attribution License, which permits unrestricted use, distribution, and reproduction in any medium, provided the original work is properly cited.

In this paper, we investigate the outage probability of per-tier in heterogeneous networks (HetNet) in the presence of aggregated interference from coexisting ad hoc networks. In ad hoc networks, a receiving node suffers the cross-tier interference signal from cellular cell and aggregated interference signal from other transmitting nodes. In order to evaluate the effect of cross-tier interference on the outage probability of ad hoc networks, we analyze the restricted region in cellular cell that the received signal interference noise ratio (SINR) of a receiving node does not exceed the SINR threshold considering the path loss model. Further, to evaluate the effect of aggregated interference of ad hoc networks, we divide the plane area into near-field area and far-field area according to whether the signal of a transmitting node could cause an outage independently or not. Based on this division, we derive the compact lower and upper boundaries of outage probability of per-tier. The computer simulations validate the results of theoretical analysis and show the effect of different factors, such as transmit power, intensity of ad hoc nodes, on the outage probability.

\section{Introduction}

The diversity of applications and devices requires several wireless networks to coexist in the same area. The networks with different technical specifications constitute a heterogeneous network [1]. Due to the extensive deployment, cellular networks are always essential in a HetNet. Some other networks with smaller coverage, such as femtocell [2] and ad hoc networks [3], are deployed in the coverage of cellular cells to improve the performance of data transmission, as shown in Figure 1. Wireless ad hoc network is a temporary network which could be deployed rapidly and conveniently without infrastructure or central node. Due to the flexible structure, it is widely applied in the areas such as military battlefield [4], emergency mission, and postdisaster reconstruction [5].

Because of the scarcity of spectrum resource, the HetNets usually share the same channels. Without the coordination between two-tier networks, the cross-tier interference is caused to each other inevitably [6]. The user equipment
(UE) in cellular networks and the receiving nodes in ad hoc networks suffer the cross-tier interference signal. In prior works, some interference coordination schemes are developed to alleviate the influence of cross-tier interference, such as spectrum allocation [7], power control [8], and interference cancellation [9].

On the other hand, the behavior of nodes in ad hoc networks is unpredicted. In a transmission interval, a subset of nodes maybe sends data simultaneously on the same channels. Therefore, a receiving node experiences the aggregated inter-node interference signal from other transmitting nodes additionally. It is evitable that the cross-tier interference and inter-node interference impair the performance of receiving nodes. In prior works [10], the effect of inter-node interference is investigated, modeling the inter-node interference as a general Poisson shot noise process [11]. While in the system that the effect of small-scale fading in wireless channel is ignored, the Poisson shot noise process model is not applicable, and the close form outage probability of a receiver could not be obtained. 


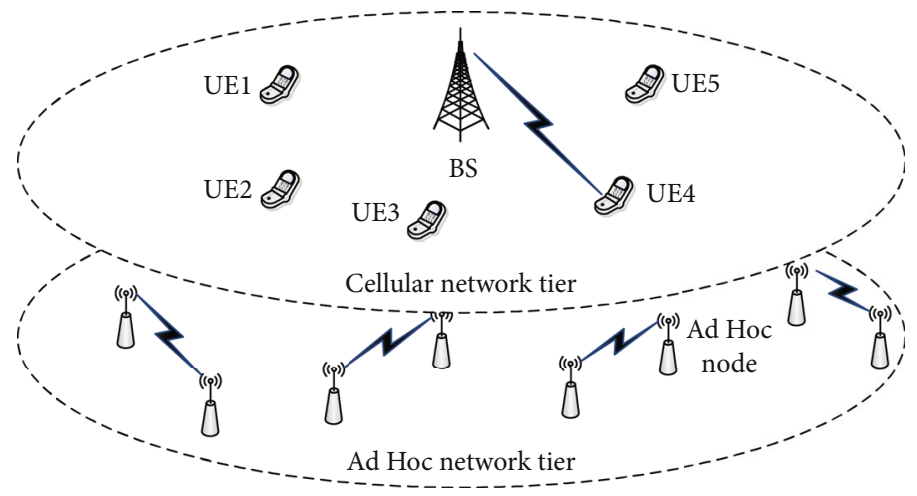

Figure 1: A HetNet consisting of cellular network and ad hoc network.

As a result, we investigate the compact boundary of outage probability in the paper. Summarily, our main contributions are given as follows.

(i) Considering the effect of cross-tier interference from cellular cell, we explore a restricted region in cellular cell that the received SINR of a receiving node does not exceed the SINR threshold.

(ii) We derive the compact lower and upper boundaries of outage probability of a receiver by dividing the plane area into near-field area and far-field area.

The remainder of the paper is structured as follows. Section 2 gives system model. Section 3 derives the compact boundary of outage probability of a receiving node. Section 4 gives the compact boundary of outage probability of a UE in cellular cell. Section 5 describes the numeric results. Finally, Section 6 gives the conclusions.

\section{System Model}

A cellular base station (BS) $B_{0}$ in the center is assumed to serve a circle region with radius $R_{c}$. The UEs locate in the coverage uniformly. In each transmission interval, the BS transmits data to a scheduling UE with power $P_{c}$. The BS and the UEs are equipped with single antenna.

In ad hoc networks, the ALOHA slotted protocol is assumed to be adopted [12]. In each slot, a subset of nodes transmits data on the same channel as cellular cell. The power of each transmitting node is assumed as $P_{f}$. Considering the nodes are placed arbitrarily, the transmitting nodes in each slot are assumed to be distributed according to a spatial Poisson point process (SPPP) with intensity $\lambda_{f}$. Hence, the set of transmitting nodes is denoted as $\Phi\left(\lambda_{f}\right)=\left\{X_{i}\right\}$, where $X_{i}$ is the position of transmitting node $i$. The model has been used, and the validity has been confirmed in prior works [13-15].

In the paper, the effect of small-scale fading of wireless channel is neglected. For the non-opportunistic scheduling system, the small-scale fading will not have a great impact on the performance. Especially for the broad-band system which adopts a robust receiver or has enough diversity gain, the effect could usually be ignored. Thus, the power of trans- mission signal decreases only with the propagation distance. For a UE or a receiving node, the receiving signal is denoted as follows

$$
P_{r}=P_{t} L D^{-\alpha}
$$

where $P_{t}$ and $P_{r}$ are powers of transmitting signal and receiving signal, respectively. $L$ is fixed loss, and $\alpha$ is path loss exponent.

\section{Outage Probability Evaluation in Ad Hoc Networks}

For a receiving node in ad hoc networks, it not only experiences the cross-tier interference signal from cellular cell but also the inter-node interference signals from other transmitting nodes. Hence, we evaluate the effect of cross-tier interference and inter-node interference on the outage probability, respectively.

3.1. Restricted Region in Cellular Cell. We assume a receiving node $k$ locates at the origin, its received SINR is denoted as

$$
\operatorname{SINR}_{f}=\frac{P_{f} L D_{f}^{-\alpha}}{P_{c} L D_{f, c}^{-\alpha}+\sum_{\varphi \in \Phi} P_{f} L\left|X_{\varphi}\right|^{-\alpha}+N_{0}}
$$

where $N_{0}$ represents the power of thermal noise. Assuming the SINR threshold of ad hoc networks is $\Gamma_{f}$, the received SINR should not be less than the threshold. Neglecting the effect of thermal noise for analysis simplicity, the distance between $\mathrm{BS} B_{0}$ and the receiving node $k$ should satisfy

$$
D_{f, c} \geq\left(\frac{P_{c}}{P_{f}} \frac{\Gamma_{f}}{D_{f}^{-\alpha}-\Gamma_{f} \sum_{\varphi \in \Phi}\left|X_{\varphi}\right|^{-\alpha}}\right)^{1 / \alpha}>\left(\frac{P_{c} \Gamma_{f}}{P_{f} D_{f}^{-\alpha}}\right)^{1 / \alpha} .
$$

Further, we assume the minimum distance between a transmitting node and its receiving node is $R$, which means the power of desired signal at receiving node could reach the maximum value. Therefore, the minimum value of $D_{f, c}$ 
is denoted as

$$
D_{f, c}^{\min }=\left(\frac{P_{c} \Gamma_{f}}{P_{f} R^{-\alpha}}\right)^{1 / \alpha}
$$

It is obvious that there is a circle area with the BS as center and with the $D_{f, c}^{\min }$ as minimum radius. According to the definition of outage probability, the outage probability of a receiving node in the area is equal to 1.

3.2. Effect of Inter-node Interference on Outage Probability. In the section, we evaluate the outage probability of a receiving node outside the mentioned area above, considering the effect of inter-node interference. The compact lower and upper boundaries of outage probability are derived.

For the receiving node $k$, the outage probability is expressed as

$$
\begin{aligned}
p_{\text {outage }}\left(D_{f}\right) & =\operatorname{Pr}\left[\operatorname{SINR}_{f}\left(D_{f}\right) \leq \Gamma_{f}\right] \\
& =\operatorname{Pr}\left(\frac{P_{f} D_{f}^{-\alpha}}{P_{c} D_{f, c}^{-\alpha}+\sum_{\varphi \in \Phi} P_{f}\left|X_{\varphi}\right|^{-\alpha}} \leq \Gamma_{f}\right) \\
& =\operatorname{Pr}\left(\sum_{\varphi \in \Phi}\left|X_{\varphi}\right|^{-\alpha} \geq \theta\right)=\operatorname{Pr}\left(E\left(\lambda_{f}\right)\right),
\end{aligned}
$$

where $\theta=P_{f} D_{f}^{-\alpha} / \Gamma_{f}-P_{c} D_{f, c}^{-\alpha}$. $\operatorname{Pr}[\cdot]$ represents the probability of an event. $E\left(\lambda_{f}\right)=\left\{\sum_{\varphi \in \Phi}\left|X_{\varphi}\right|^{-\alpha} \geq \theta\right\}$ represents outage event.

In case that the distances in desired signal and cross-tier interference signal are fixed, equation (5) indicates the outage probability is decided by the distribution of ad hoc nodes. For the receiving node $k$, the nearby transmitting nodes contribute to the major interference. Hence, with the receiving node $k$ as the center, we divide the plane space into two areas: near-field area and far-field area, as shown in Figure 2. The definitions of near-field area and far-field area are given as follows.

Definition 1. Near-field area $Z_{n}=c(o, s)$ is a circle area with receiving node $k$ as the center and $s(s>0)$ as the radius. The set of transmitting nodes in $Z_{n}$ is denoted as $\Phi_{n}=\Phi \cap Z_{n}$, in which the interference signal from any transmitting node could cause an outage of receiving node $k$.

In terms of definition 1, the selection of the radius of near-field area should ensure one or several transmitting nodes in the area. This means that the radius $s$ of nearfield area should satisfy $s^{-\alpha} \geq \theta$, i.e., $s \leq \theta^{-1 / \alpha}$.

Definition 2. Far-field area includes the plane area other than the near-filed area, denoted as $Z_{o}=\mathbb{R}^{2} \backslash Z_{n}=\bar{c}(o, s)$. The set of transmitting nodes in $Z_{o}$ is denoted as $\Phi_{o}=\Phi \backslash \Phi_{n}$, in which the aggregated interference signal from multiple transmitting nodes could cause an outage of receiving node $k$.

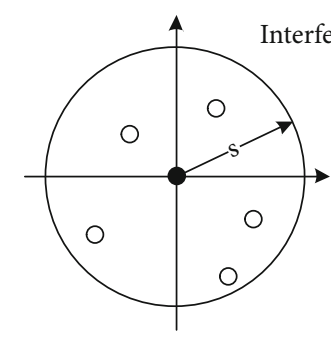

Near-field area
Far-field area

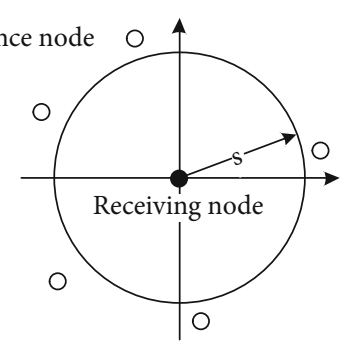

Figure 2: The division of near-field area and far-field area.

Definition 3. The event that the aggregated interference signal from the transmitting nodes in near-field area or farfield area cause an outage of receiving node $k$ is defined as event $E_{n}\left(\lambda_{f}, s\right)$ or $E_{o}\left(\lambda_{f}, s\right)$. The events are denoted as

$$
\begin{gathered}
E_{n}\left(\lambda_{f}, s\right)=\left\{\sum_{\varphi \in \Phi_{n}}\left|X_{\varphi}\right|^{-\alpha} \geq \theta\right\}, \\
E_{o}\left(\lambda_{f}, s\right)=\left\{\sum_{\varphi \in \Phi_{o}}\left|X_{\varphi}\right|^{-\alpha} \geq \theta\right\} .
\end{gathered}
$$

According to definition 3, we further define an event as $E_{s}\left(\lambda_{f}, s\right)=E_{n}\left(\lambda_{f}, s\right) \cup E_{o}\left(\lambda_{f}, s\right)$. In case $s \leq \theta^{-1 / \alpha}$, we have $E_{n}$ $\left(\lambda_{f}, s\right) \subset E\left(\lambda_{f}\right) \subseteq E_{s}\left(\lambda_{f}, s\right)$.

3.2.1. Compact Lower Boundary of Outage Probability. Due to $E_{n}\left(\lambda_{f}, s\right) \subset E\left(\lambda_{f}\right)$, then, we have

$$
p_{\text {outage }}\left(D_{f}\right)=\operatorname{Pr}\left[E\left(\lambda_{f}\right)\right]>\operatorname{Pr}\left[E_{n}\left(\lambda_{f}, s\right)\right] .
$$

Hence, the compact lower boundary of outage probability is the maximum of $\operatorname{Pr}\left[E_{n}\left(\lambda_{f}, s\right)\right]$. When the event $E_{n}\left(\lambda_{f}, s\right)$ occurs, there are one or several transmitting nodes in near-field area, thus, the event $E_{n}\left(\lambda_{f}, s\right)=\left\{\Phi \cap Z_{n} \neq \varnothing\right\}$. Considering the transmitting nodes in ad hoc networks are distributed as a SPPP with intensity $\lambda_{f}$, the probability of the event $E_{n}\left(\lambda_{f}, s\right)$ is denoted as

$$
\begin{aligned}
\operatorname{Pr}\left[E_{n}\left(\lambda_{f}, s\right)\right] & =\operatorname{Pr}\left[\Phi \cap Z_{n} \neq \varnothing\right]=1-\operatorname{Pr}[\Phi \cap c(o, s)=\varnothing] \\
& =1-\exp \left(-\pi \lambda_{f} s^{2}\right)
\end{aligned}
$$

where $s \in\left(0, \theta^{-1 / \alpha}\right]$.

Note that the probability of the event $E_{n}\left(\lambda_{f}, s\right)$ is monotonically increasing at the radius $s$. Therefore, the maximum value of $\operatorname{Pr}\left[E_{n}\left(\lambda_{f}, s\right)\right]$ which is the compact lower boundary of outage probability could be obtained when $s=\theta^{-1 / \alpha}$, denoted as

$$
p_{\text {outage }}^{\text {lower }}\left(D_{f}\right)=1-\exp \left(-\pi \lambda_{f} \theta^{-2 / \alpha}\right)
$$


3.2.2. Compact Upper Boundary of Outage Probability. Due to $E\left(\lambda_{f}\right) \subseteq E_{s}\left(\lambda_{f}, s\right)$, then, we have

$$
p_{\text {outage }}\left(D_{f}\right)=\operatorname{Pr}\left[E\left(\lambda_{f}\right)\right] \leq \operatorname{Pr}\left[E_{s}\left(\lambda_{f}, s\right)\right]
$$

Hence, the compact upper boundary of outage probability is the minimum of $\operatorname{Pr}\left[E_{n}\left(\lambda_{f}, s\right)\right]$. Considering $E_{s}\left(\lambda_{f}, s\right)$ $=E_{n}\left(\lambda_{f}, s\right) \cup E_{o}\left(\lambda_{f}, s\right)$, the probability of the event $E_{s}\left(\lambda_{f}, s\right)$ is expressed as

$$
\begin{aligned}
\operatorname{Pr}\left[E_{s}\left(\lambda_{f}, s\right)\right]= & \operatorname{Pr}\left[E_{n}\left(\lambda_{f}, s\right) \cup E_{o}\left(\lambda_{f}, s\right)\right] \\
= & \operatorname{Pr}\left[E_{n}\left(\lambda_{f}, s\right)\right]+\operatorname{Pr}\left[E_{o}\left(\lambda_{f}, s\right)\right] \\
& -\operatorname{Pr}\left[E_{n}\left(\lambda_{f}, s\right)\right] \operatorname{Pr}\left[E_{o}\left(\lambda_{f}, s\right)\right] \\
= & \operatorname{Pr}\left[E_{n}\left(\lambda_{f}, s\right)\right]+\operatorname{Pr}\left[E_{o}\left(\lambda_{f}, s\right)\right] \\
& \cdot\left\{1-\operatorname{Pr}\left[E_{n}\left(\lambda_{f}, s\right)\right]\right\} \\
= & 1-\left\{1-\operatorname{Pr}\left[E_{n}\left(\lambda_{f}, s\right)\right]\right\} \\
& \cdot\left\{1-\operatorname{Pr}\left[E_{o}\left(\lambda_{f}, s\right)\right]\right\} .
\end{aligned}
$$

In equation (12), the event $E_{n}\left(\lambda_{f}, s\right)$ and $E_{o}\left(\lambda_{f}, s\right)$ are independent of each other. When the radius $s$ changes, $\operatorname{Pr}\left[E_{n}\left(\lambda_{f}, s\right)\right]$ increases but $\operatorname{Pr}\left[E_{o}\left(\lambda_{f}, s\right)\right]$ decreases, or vice versa [16]. Hence, in order to obtain the compact upper boundary of outage probability, the selection of $s$ should satisfy $\operatorname{Pr}\left[E_{n}\left(\lambda_{f}, s\right)\right]=\operatorname{Pr}\left[E_{o}\left(\lambda_{f}, s\right)\right]$ to make $\operatorname{Pr}\left[E_{s}\left(\lambda_{f}, s\right)\right]$ to be minimum.

Note that the close form of $\operatorname{Pr}\left[E_{o}\left(\lambda_{f}, s\right)\right]$ could not be derived, thus, we adopt the Chebyshev boundary to replace it. To obtain the Chebyshev boundary, we introduce two theorems as follows.

Theorem 4 (Campbell formula). Let $\Phi$ is a stationary Poisson point process of intensity $\lambda$ on $\mathbb{R}^{2}$, then, for a nonnegative function $f, \sum_{X \in \Phi} f(X)$ is a random variable with mean value and variance as follows

$$
\begin{array}{r}
\mathbb{E}\left[\sum_{X \in \Phi} f(X)\right]=\lambda \int_{\mathbb{R}^{d}} f(x) d x, \\
\operatorname{var}\left[\sum_{X \in \Phi} f(X)\right]=\lambda \int_{\mathbb{R}^{d}} f^{2}(x) d x .
\end{array}
$$

Theorem 5 (Chebyshev inequality). Let $X$ is a random variable, and its mean value $\mu=\mathbb{E}[|X|]$ and variance $\sigma^{2}=\operatorname{var}[X]$. For any real number $c>0$,

$$
\operatorname{Pr}(|X-\mu| \geq c) \leq \frac{\sigma^{2}}{c^{2}}
$$

According to theorem 4, the mean value and variance of the aggregated interference from the nodes in far-field area $I_{o}=\sum_{\varphi \in \Phi_{o}}\left|X_{\varphi}\right|^{-\alpha}$ could be obtained when $\alpha>2$, denoted as

$$
\begin{gathered}
\mu=\mathbb{E}\left[\sum_{\varphi \in \Phi_{o}}\left|X_{\varphi}\right|^{-\alpha}\right]=2 \pi \lambda_{f} \int_{s}^{\infty} r^{-\alpha} r \mathrm{~d} r=\frac{2 \pi \lambda_{f}}{\alpha-2} s^{2-\alpha}, \\
\sigma^{2}=\operatorname{var}\left[\sum_{\varphi \in \Phi_{o}}\left|X_{\varphi}\right|^{-\alpha}\right]=2 \pi \lambda_{f} \int_{s}^{\infty} r^{-2 \alpha} r \mathrm{~d} r=\frac{\pi \lambda_{f}}{\alpha-1} s^{2(1-\alpha)} .
\end{gathered}
$$

Further, in terms of theorem 5, the Chebyshev boundary of $\operatorname{Pr}\left[E_{o}\left(\lambda_{f}, s\right)\right]$ is denoted as

$$
\operatorname{Pr}\left[E_{o}\left(\lambda_{f}, s\right)\right] \leq \operatorname{Pr}\left(\frac{\left|I_{o}-\mu\right|}{\theta-\mu} \geq 1\right) \leq \frac{\sigma^{2}}{(\theta-\mu)^{2}}=\operatorname{Pr}^{\text {upper }}\left[E_{o}\left(\lambda_{f}, s\right)\right]
$$

In case the outage probability is limited by a strict quantity of service (QoS) threshold, the intensity of interference nodes shall be very small. Hence, when $\lambda_{f}$ tends to 0 , $\operatorname{Pr}^{\text {upper }}\left[E_{o}\left(\lambda_{f}, s\right)\right]$ is approximated as

$$
\operatorname{Pr}^{\text {upper }}\left[E_{o}\left(\lambda_{f}, s\right)\right]=\frac{\pi \lambda_{f}}{(\alpha-1) \theta^{2}} s^{2(1-\alpha)}+\Theta\left(\lambda_{f}^{2}\right) \text {. }
$$

Note that the probability of the event $E_{n}\left(\lambda_{f}, s\right)$ is derived in equation (9). Hence, by making $\operatorname{Pr}\left[E_{n}\left(\lambda_{f}, s\right)\right]=\operatorname{Pr}^{\text {upper }}[$ $\left.E_{o}\left(\lambda_{f}, s\right)\right]$ and discarding the high order term $\Theta\left(\lambda_{f}^{2}\right)$, the optimal value of $s$ could be obtained. When $\lambda_{f}$ tends to 0 , we have

$$
s=\theta^{-1 / \alpha}(\alpha-1)^{-1 / 2 \alpha} .
$$

Substituting the results in equations (9), (19), and (20) into equation (12), the minimum value of $\operatorname{Pr}\left[E_{s}\left(\lambda_{f}, s\right)\right]$, i.e., the compact upper boundary of outage probability is denoted as

$$
p_{\text {outage }}^{\text {upper }}\left(D_{f}\right)=1-\exp \left(-\frac{2 \pi \lambda_{f}}{\theta^{2 / \alpha}(\alpha-1)^{1 / \alpha}}\right)
$$

\section{Outage Probability Evaluation in Cellular Cell}

In cellular cell, a UE experiences the aggregated cross-tier interference signal from ad hoc networks in downlink transmissions. The outage probability of a UE at the origin is denoted as

$$
\begin{aligned}
P_{\text {outage }}\left(D_{c}\right) & =\operatorname{Pr}\left[\operatorname{SINR}_{c}\left(D_{c}\right) \leq \Gamma_{c}\right]=\operatorname{Pr}\left(\frac{P_{c} D_{c}^{-\alpha}}{\sum_{\varphi \in \Phi} P_{f}\left|X_{\varphi}\right|^{-\alpha}} \leq \Gamma_{c}\right) \\
& =\operatorname{Pr}\left(\sum_{\varphi \in \Phi}\left|X_{\varphi}\right|^{-\alpha} \geq \vartheta\right),
\end{aligned}
$$




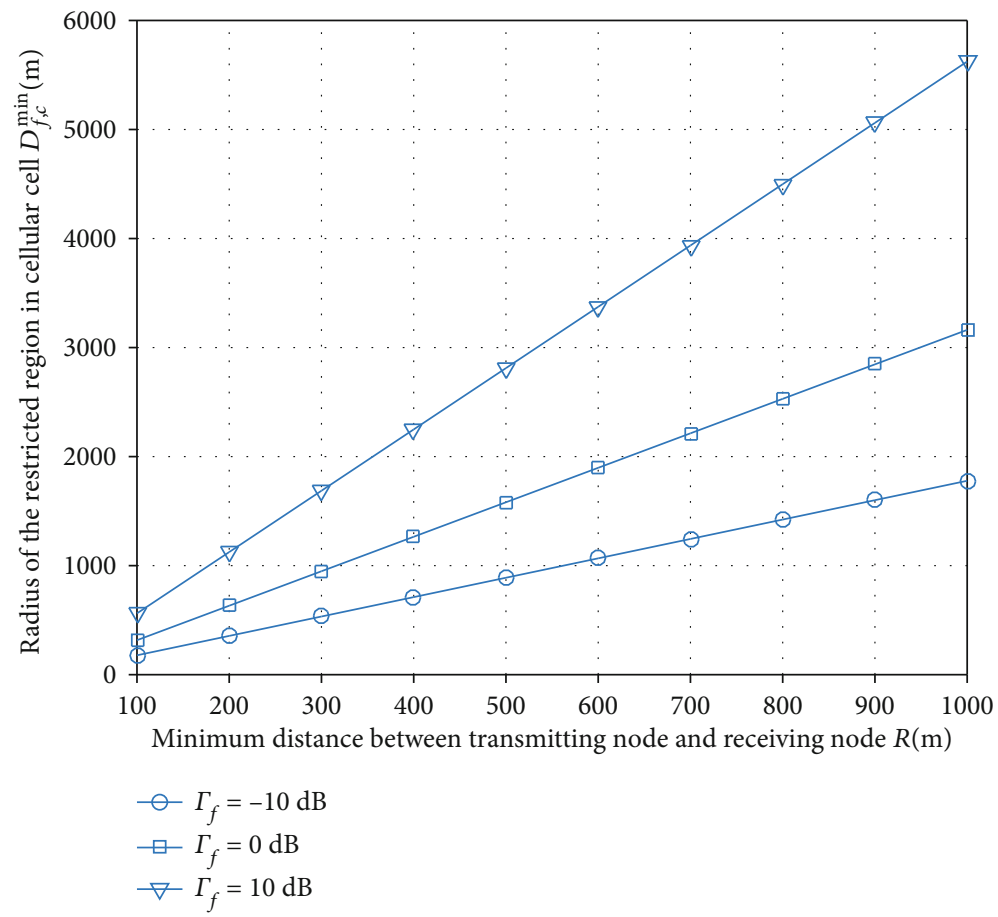

FIGURE 3: Radius of the restricted region vs. minimum distance between transmitting node and receiving node.

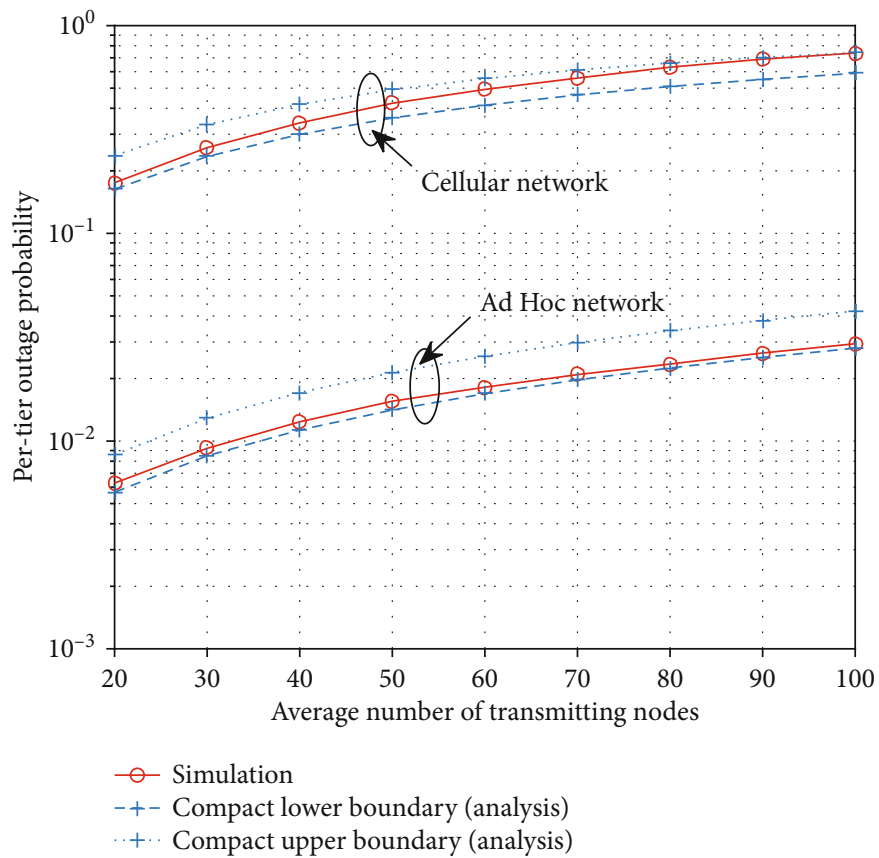

FIGURE 4: Per-tier outage probability vs. average number of transmitting nodes.

where $\vartheta=P_{c} D_{c}^{-\alpha} / P_{f} \Gamma_{c}$. We note that the outage probability of a UE in equation (22) has the same expression as that in equation (5). Thus, according to the derivation in Sections 3.2.1 and 3.2.2, we could obtain the compact lower and upper boundaries of outage probability by replacing $\theta$ in equations (10) and (21) with $\vartheta$, respectively.

\section{Numeric Results}

In the section, we give the numeric results from computer simulations. In the simulations, the transmit power and service radius of cellular BS are assumed to be $P_{c}=46 \mathrm{dBm}$ and $R_{c}=1000 \mathrm{~m}$, respectively. The transmit power of ad hoc node 


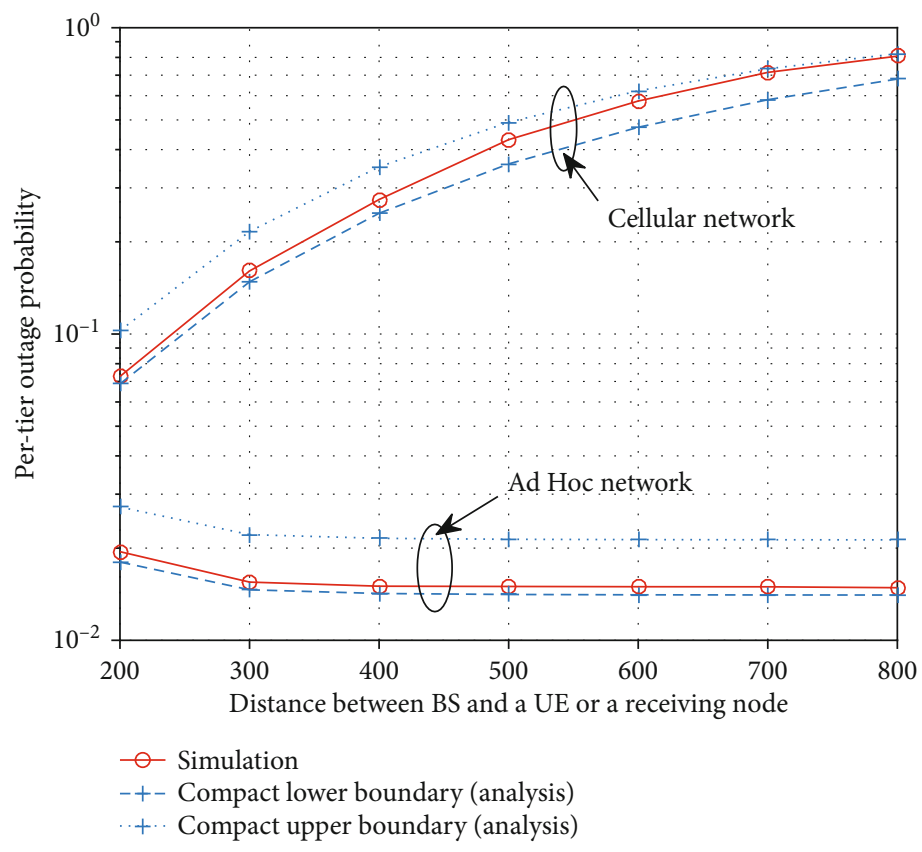

FIGURE 5: Per-tier outage probability vs. distance between BS and a UE or a receiving node.
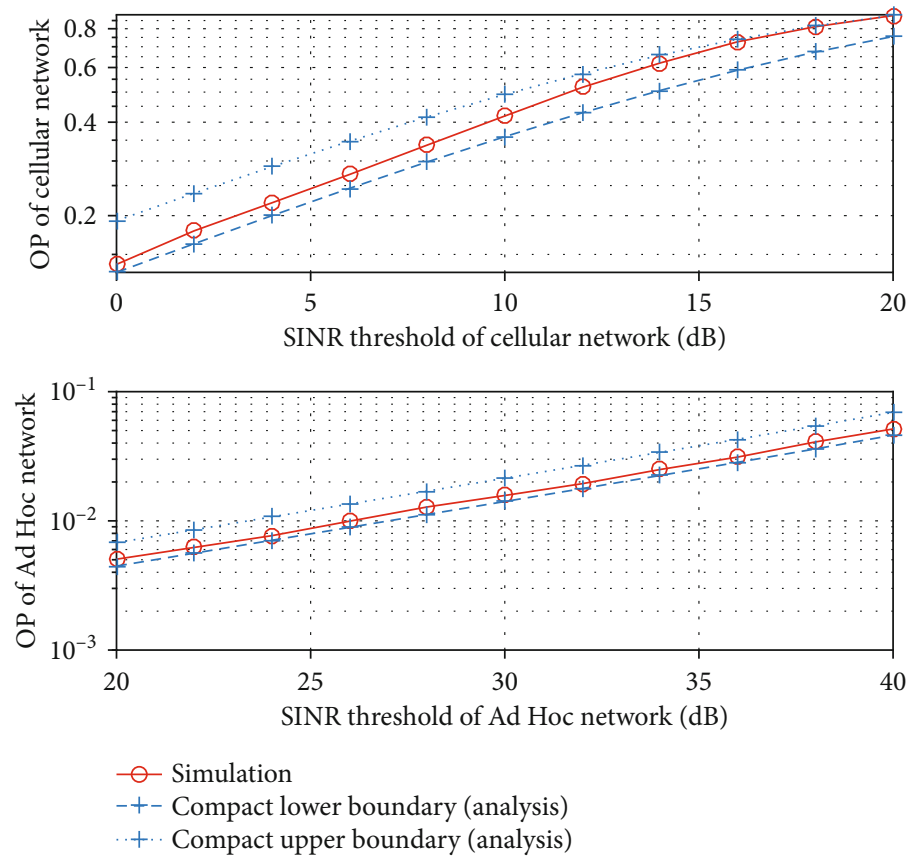

FIgUre 6: Per-tier outage probability vs. SINR threshold.

is assumed to be $P_{f}=23 \mathrm{dBm}$, and the path loss exponent is $\alpha=4$. The other parameters vary in the simulations.

Figure 3 shows the relation between the radius of the restricted region and the minimum distance between transmitting node and receiving node. The increase of minimum distance decreases the power of desired signal and the received SINR of a receiving node. Hence, the restricted region in which the received SINR could not exceed the SINR threshold becomes larger. On the other hand, with the increase of SINR threshold, the received SINR of more receiving node could not exceed the SINR threshold. The nodes should to be contained in the restricted region. Hence, the radius of restricted region increases.

Figure 4 reflects the effect of the number of transmitting nodes on per-tier outage probability. The increase of the transmitting nodes causes a growth of the power of crosstier interference or inter-node interference. With the fixed desired signal and SINR threshold, the received SINR decreases but the outage probability increases. Moreover, the reception of a receiving node is influenced by cross-tier 
interference and inter-node interference simultaneously. The increase of transmitting nodes only leads to the growth of the power of inter-node interference. Hence, the outage probability of ad hoc networks increases relatively slowly with the increase of the transmitting nodes.

Figure 5 depicts the change of outage probability with the distance between BS and a UE or a receiving node. For a UE, the power of desired signal decreases with the increase of distance far from the BS. Hence, the received SINR decreases but the outage probability increases. While for a receiving node, the increase of distance reduces the power of cross-tier interference. With the fixed desired signal and SINR threshold, the received SINR increases but the outage probability decreases. However, when the distance increases to a certain value, the outage probability of a receiving node is mainly affected by inter-node interference, thus, the outage probability is almost constant with the increase of the distance.

In Figure 6, the results that the outage probability changes with SINR threshold are given. No matter what the receiver is a UE or a receiving node, the results indicate the promotion of SINR threshold causes an increase of outage probability.

\section{Conclusions}

The paper investigates the outage probability of per-tier in HetNet in the presence of aggregated interference from coexisting ad hoc networks. The restricted region in cellular cell that the received SINR of a receiving node does not exceed the SINR threshold is explored to evaluate the effect of cross-tier interference. Further, to evaluate the effect of aggregated interference of ad hoc networks, the plane area is divided into near-field area and far-field area according to whether the signal of a transmitting node could cause an outage independently or not. Based on this division, the compact lower and upper boundaries of outage probability of per-tier are derived. The computer simulations validate the results of theoretical analysis.

We admit there are some limitations in the paper. For instance, the small-scale fading of wireless channel is not taken into account. As a future work, we will explore the performance optimization by interference management or resource assignment based on the derived results.

\section{Data Availability}

Some or all data, models, or code generated or used during the study are available from the corresponding author by request.

\section{Conflicts of Interest}

The authors declare that they have no conflicts of interest.

\section{References}

[1] Y. Zhong, T. Q. S. Quek, and X. Ge, "Heterogeneous cellular networks with spatio-temporal traffic: delay analysis and scheduling," IEEE Journal on Selected Areas in Communications, vol. 35, no. 6, pp. 1373-1386, 2017.

[2] R. Ahmad, E. A. Sundararajan, and A. Khalifeh, "A survey on femtocell handover management in dense heterogeneous $5 \mathrm{G}$ networks," Telecommunication System, vol. 75, no. 4, pp. 481-507, 2020

[3] A. Scaglione, D. L. Goeckel, and J. N. Laneman, "Cooperative communications in mobile ad hoc networks," IEEE Signal Processing Magazine, vol. 23, no. 5, pp. 18-29, 2006.

[4] P. Phaswana and M. Velempini, "Paper spectrum-aware transitive multicast on demand distance vector routing for military cognitive radio ad hoc networks," in 2019 IEEE AFRICON, Accra, Ghana, September 2019.

[5] J. Liu and N. Kato, "A Markovian analysis for explicit probabilistic stopping-based information propagation in postdisaster ad hoc mobile networks," IEEE Transactions on Wireless Communications, vol. 15, no. 1, pp. 81-90, 2016.

[6] S. Lee, S. Kim, Y. Park, S. Choi, and D. Hong, "Effect of unpredictable interference on MU-MIMO systems in HetNet," IEEE Access, vol. 6, pp. 28870-28876, 2018.

[7] J. Dai, W. Lv, and F. Ye, "Spectrum allocation scheme based on Stackelberg game in two-tier HetNet," in 2016 IEEE 13th International Conference on Signal Processing (ICSP), pp. 12701274, Chengdu, China, November 2016.

[8] Hae Beom Jung and Duk Kyung Kim, "Power control of femtocells based on max-min fairness in heterogeneous networks," IEEE Communication Letter, vol. 17, no. 7, pp. 13721375, 2013.

[9] K. Song, B. Ji, Y. Huang, M. Xiao, and L. Yang, "Performance analysis of heterogeneous networks with interference cancellation," IEEE Transaction on Vehicle Technology, vol. 66, no. 8, pp. 6969-6981, 2017.

[10] W. Zhang and J. Wang, "Topology control with outage probability constraint in wireless ad hoc networks," Journal of Computers, vol. 32, no. 3, pp. 86-98, 2021.

[11] F. BaccelliAnup and B. Biswas, "On scaling limits of power law shot-noise fields," Stochastic Models, vol. 31, no. 2, pp. 1-17, 2014.

[12] B. Blaszczyszyn, P. Mühlethaler, and Y. Toor, "Stochastic analysis of ALOHA in vehicular ad hoc networks," Annals of Telecommunications, vol. 68, no. 1-2, pp. 95-106, 2013.

[13] J. Kingman, Poisson Processes, Oxford University Press, London, U.K., 1993.

[14] V. Chandrasekhar, M. Kountouris, and J. G. Andrews, "Coverage in multi-antenna two-tier networks," IEEE Transactions on Wireless Communications, vol. 8, no. 10, pp. 5314-5327, 2009.

[15] M. Haenggi, J. G. Andrews, F. Baccelli, O. Dousse, and M. Franceschetti, "Stochastic geometry and random graphs for the analysis and design of wireless networks," IEEE Journal on Selected Areas in Communications, vol. 27, no. 7, pp. 10291046, 2009.

[16] S. P. Weber, X. Yang, J. G. Andrews, and G. DeVeciana, "Transmission capacity of wireless ad hoc networks with outage constraints," IEEE Transactions on Information Theory, vol. 51, no. 12, pp. 4091-4102, 2005. 